\title{
Probing collective effects in hadronisation with the extremes of the underlying event
}

\author{
T. Martin ${ }^{1, a}$, P. Skands ${ }^{2}$, S. Farrington ${ }^{1}$ \\ ${ }^{1}$ Department of Physics, University of Warwick, Coventry CV47AL, UK \\ 2 School of Physics and Astronomy, Monash University, Clayton, VIC 3800, Australia
}

Received: 24 March 2016/ Accepted: 7 May 2016 / Published online: 25 May 2016

(C) The Author(s) 2016. This article is published with open access at Springerlink.com

\begin{abstract}
We define a new set of observables to probe the structure of the underlying event in hadron collisions. We use the conventional definition of the "transverse region" in jet events and, for a fixed window in jet $p_{\perp}$, propose to measure several discriminating quantities as a function of the level of activity in the transverse region. The measurement of these observables in LHC data would reveal whether, e.g., the properties of "low-UE" events are compatible with equivalent measurements in $e^{+} e^{-}$collisions (jet universality), and whether the scaling behaviour towards "high-UE" events exhibits properties of non-trivial soft-QCD dynamics, such as colour reconnections or other collective phenomena. We illustrate at $\sqrt{s}=13 \mathrm{TeV}$ that significant discriminatory power is obtained in comparisons between MC models with varying treatments of collective effects, including PYTHIA 8 , EPOS, and DIPSY.
\end{abstract}

\section{Introduction}

The "jet pedestal effect", now called the underlying event (UE), was first observed by the UA1 experiment at CERN's Sp $\bar{p} S$ collider, in an early study of jet events [1]. This study concluded that, outside the core of a jet, "a constant $E_{\mathrm{T}}$ plateau is observed, whose height is independent of the jet $E_{\mathrm{T}}$ " [1]. The plateau was substantially higher than that observed in minimum-bias events ${ }^{1}$ at the same energy [3-6]. Its characteristics have since been studied extensively, in $p p$ and $p \bar{p}$ collisions over two orders of magnitude in Centre of Mass (CM) energy, from RHIC [7,8] through Tevatron [912] to LHC energies [13-25], the latter most recently extending to measurements at $13 \mathrm{TeV}$ [26].

Theoretically, the UE is often roughly defined as the collection of particles produced in a single hadronic inter-

\footnotetext{
${ }^{1}$ For a definition of the term minimum-bias, see e.g. [2, Chp. 7].

a e-mail: tim.martin@ cern.ch
}

action which do not originate from the primary ("hard") parton-parton scattering, nor relate directly to it in the form of hadronised parton showers. However, since the hardinteraction initiators are coloured (quarks or gluons), confinement implies that it is in general not possible to define uniquely which hadrons were produced by the hard interaction and which belong to the UE.

Instead, physical observables that measure phase-space regions "outside the core of jets" are used to provide operational definitions of the UE. Two main definitions are in current use: the most common one is a purely geometrical definition of the UE as an angular region transverse to a leading charged particle or jet [9], typically spanning $90^{\circ} \pm 30^{\circ}$; an alternative definition of the UE is what remains when hard jets have been removed, an early example of this being the "Swiss Cheese" method introduced by CDF [10] now superseded by so-called "jet median/area" techniques proposed in [27] and used e.g. by CMS [19,28].

In addition to the average UE properties, several studies at the LHC have now also measured observables sensitive to the per-event fluctuations. These include the ATLAS [14] study of the widths of the charged-particle density and scalar$p_{\perp}$ sum distributions, and the CMS [22] study of jet multiplicity in the UE. Such studies are crucial to pin down the underlying physics mechanisms, revealing e.g. potentially interesting deviations from Poissonian fluctuations, and to what extent (soft) jets are required to build up the UE activity. In this paper, we propose to go a step further, and study the UE properties as a function of its in-situ level; this will allow a detailed exploration of the evolution of quantities such as strangeness fractions, particle spectra, and (soft) jet rates, from low to high underlying activity. Though we focus mainly on identified-particle rates, we emphasise that the method is general and can be applied to any UE property as a function of any measure of UE activity. The explicit studies we propose are inspired by - and are intended to be complementary to-minimum-bias studies as a function of multi- 
plicity. Of special interest is whether events with very low UE levels exhibit particle yields and spectra more consistent with LEP fragmentation models than those of their higherUE counterparts, and whether high-UE events exhibit any clear signs of flow or other collective effects. A complementary recent interesting proposal is that of [29] which suggests to measure similar observables using forward multiplicity as the indicator of event activity.

On the theory side, the fragmentation process in the simple reference case of hadronic $Z$ decays is believed to be relatively well understood, and shower+hadronisation models such as those implemented in the general-purpose Monte Carlo models HeRWIG [30], PYTHIA [31], and SHERPa [32], are capable of describing the majority of $e^{+} e^{-} \rightarrow$ hadrons data well (see e.g. the plots available on mcplots [33]). However, moving to $p p$ collisions, several additional complications arise, including initial-state radiation, beam remnants, diffraction, and multiple parton interactions (MPI). The corresponding physics models are therefore necessarily also more complicated. Starting from Rick Field's observation with his "Tune A" [34] of the PYTHIA 6 MPI model $[35,36]$ to Tevatron data [10] that a much higher degree of colour correlation than anticipated (between partons from different MPI systems) was required to fit the $p_{\perp}$ spectra of particles in the UE at CDF, there have appeared progressively more indications that the physics of hadron collisions is more complicated than was previously thought. The clues include the increase of the average $p_{\perp}$ with event multiplicity [35,37-42], the large yields of hyperons [43-50], and the overall relatively steep increase of average $p_{\perp}$ with hadron mass [45,46,49-54] observed in $p p$ collisions. There are also more subtle indications, such as the unexpected ridge-like structure in two-particle correlations at low $\Delta \phi$ in high-multiplicity minimum-bias $p p$ collisions observed by CMS and ATLAS $[55,56]$. To shed further light on these effects, many studies of minimum-bias events now focus on the scaling with charged-particle multiplicity (see e.g. [29,41,42,57-59]), with a special emphasis on the highest accessible multiplicities. Here we extend this type of study to the UE context.

At central rapidities, particle production in the UE is predominantly driven by the hadronisation of soft/semi-hard secondary scatters within the colliding hadrons. The presence of the requirement for high- $p_{\perp}$ activity, such as that provided by a (hard) experimental trigger, biases the UE towards larger activities than that of more inclusive (e.g. minimumbias) event samples. Within the context of MPI models (see e.g. [2, Chp. 7]), this can be understood as a consequence of a bias towards smaller impact parameters, with the onset of the aforementioned UE plateau signalling that the average impact parameter has become significantly smaller than the radius of the proton $\langle b\rangle \ll r_{p}$, such that the two matter distributions are already completely overlapping from that point onward (see e.g. [35, sect. VI.D]). There are, however, still large fluctuations expected, some geometric in origin, others dynamic. Examples of geometric fluctuations include, for instance, the rare case of a large-impact parameter interaction producing a hard jet presumably accompanied by very little UE, or the case of two protons happening to be compressed transversely producing a larger-than-ordinary matter overlap and hence a very large UE. Even for fixed matter overlap, the number and hardness of MPI and shower branchings will exhibit random (quantum) fluctuations, and especially the tail towards high activities may be sensitive to new QCD dynamics. Currently, we are not aware of any single phenomenological model that spans all the possibilities. Nevertheless, on general grounds, we expect baryon and strangeness fractions to be good tracers of the underlying mechanisms (in addition to hard MPI rates):

- The strange quark, having a mass very near $\Lambda_{\mathrm{QCD}}$, can act as a sensitive probe of any changes to the energy density available at the time of hadronisation. Simply put, the more strange quarks we see, relative to $u$ and $d$ quarks, the higher the effective string tension or effective temperature we would extract, depending on the model. Note, however, that the following two kinematic effects can also affect the interpretation of strangeness ratios: firstly, the energy cost of producing a strange-quark rest mass, as compared to say an up or down one, is relatively more significant for a hadronising system with small invariant mass than for one with large invariant mass; thus we expect small-mass systems to exhibit lower strangeness ratios simply due to phase-space restrictions. Secondly, in the presence of cuts on $p_{\perp}$, the modelling of $p_{\perp}$ spectra affects the ratio of the observed to total strange production.

- Baryon production probes a unique colour topology and has clear experimental signatures. Unlike mesons, baryons do not appear naturally in the "leading-colour" approximation $\left(N_{C} \rightarrow \infty\right)$, which is so heavily relied upon by $\mathrm{MC}$ event generators. This makes their production somewhat more difficult to model and interpret but also more interesting as a probe of aspects beyond leading $N_{C}$. The dependence on mass and strangeness can be studied in detail, especially via dedicated Particle Identification (PID) capabilities. For example, a recent ALICE study managed to identify and study strongly decaying excited states such as $\Sigma(1385)^{ \pm}$and $\Xi(1530)^{0}[50]$.

Combined, strangeness, baryon number, and mass (or spinmultiplet) constitute a powerful 3-dimensional ladder along which to chart the behaviour of the underlying event. All of the major LHC experiments can provide sensitive measurements for key quantities such as multiplicities of charged tracks, $K_{\mathrm{s}}^{0}$, and $\Lambda$, while the more specialised PID capabili- 
ties of the ALICE and LHCb experiments permit reconstruction of several of the more challenging (e.g. multi-strange and excited) states that will be needed to complete the picture. The production of each particle species can then be understood in the context of the overall modelling. For instance, a measurement concluding that the production of kaons is low in a given model can have vastly different physics implications, depending on whether the model underpredicts the rates of all other particles as well.

In this study, the most powerful model discrimination arises from taking the ratios of averaged identified-particle yields as described in Sect. 3. This highlights the salient physics while minimising the effects from global scaling differences between the considered models.

Though we focus on central rapidities in this paper, we round off by noting that, at forward rapidities, the particle production is increasingly sensitive to the hadronisation of the beam remnants, and to diffraction. In the context of MPI models, the amount of energy (both total and transverse) scattered into the forward direction is also sensitive to the shape of parton distribution functions (PDFs) at low $x$ (see e.g. [60]). Thus measurements at forward rapidities (e.g. AFP, ALFA, LHCb, LHCf, TOTEM) are uniquely sensitive to these aspects and are required to understand the full dynamics. An obvious example of interest is the colour structure of the beam remnant, which is tied in with that of the MPI by overall colour conservation. ${ }^{2}$ Studying the fragmentation process for diffractively tagged events would also furnish insights complementary to those provided by the measurements we propose here.

\section{Monte Carlo models}

For this study, we include the set of MC event generators and tunes listed in Table 1. This permits us to span a significant range of possible dynamic effects, including colour ropes (with DIPSY), hydrodynamics (with EPOS), and colour reconnections ("CR", with PYTHIA 8). The varying dynamical assumptions imply that these models will generate qualitatively different predictions for the scaling with transverse activity in one or more of the observables we consider below. In all cases, an inclusive 'minimum bias' event sample was generated for analysis at $\sqrt{s}=13 \mathrm{TeV}$. The key features of these models, in the context of this paper, are described below. 10 million events were simulated for each sample. The analysis was performed with RIVET [61], including the use of FASTJET [62].

\footnotetext{
2 Since the colliding hadrons are colour neutral, the colour charge of each hadron remnant must be equal and opposite (in phase) to the sum of those of the MPI initiators (including the hardest-interaction initiators) taken from that hadron.
}

Table 1 MC generators, versions and tunes used in this paper.

\begin{tabular}{lll}
\hline & Version & Tune \\
\hline DIPSY & ThePEG++ 2015-08-11 & NoSwing \\
DIPSY & ThePEG++ 2015-08-11 & Rope \\
EPOS & 1.3 & LHC \\
PYTHIA 8 & 8.210 & Monash \\
PYTHIA 8 & 8.210 & Monash + new CR \\
\hline
\end{tabular}

\subsection{Pythia and colour reconnections}

The description of soft-inclusive physics in PYTHIA is rooted in perturbation theory, starting from a resummed (unitarised) picture of multiple perturbative parton-parton interactions (MPI) [35] supplemented by (interleaved) $p_{\perp}$-ordered parton showers [63]. This builds up a partonic substructure which can act as the starting point for the non-perturbative modelling of each event. ${ }^{3}$ However, when there is more than one MPI the perturbative era does not completely fix the detailed colour structure of the beam remnant, nor does it fix the colour correlations between individual MPI systems [64]. Additionally, within each system, ambiguities beyond leading colour could affect the formation of strings, and strings could conceivably even interact dynamically in the limited time between formation and fragmentation. Recent years have therefore seen increased explorations of new alternatives though so far only a part of the full range of possibilities have been explicitly addressed $[65,66]$.

The default modelling of colour flow in PYTHIA 8 [67], which is the one used by the baseline Monash tune [60], is as follows: the partons of each MPI system are either allowed to form their own "skeleton" in colour space or, with a usermodifiable probability, they are merged with the colour structure of a higher- $p_{\perp}$ MPI system. In the latter case, each parton from the lower- $p_{\perp}$ system is merged onto a string piece of the higher- $p_{\perp}$ system where it will cause the least increase in total string length. Within the context of this model, the minimisation of string length is likely to be a physically reasonable dynamical principle, representing potential-energy minimisation, but there is no a priori basis for guessing precisely what reconnection probability to choose, nor whether it should be constant at all CM energies.

More recently, a model that attempts to anchor itself more firmly in QCD was proposed [66], which we here label "Monash + New CR" (cf. Table 1). It uses an approximation to the full group-theoretical weights from $\mathrm{SU}(3)$ to compute the probabilities for alternative string topologies at the subleading- $N_{C}$ level, and is again combined with a dynamical selection favouring topologies with low string lengths. A

\footnotetext{
${ }^{3}$ Exceptions are elastic and low-mass diffractive scatterings, which are modelled without a perturbative era.
} 
novel feature of this model is that it allows for "string junctions" (colour-epsilon and -anti-epsilon structures) to form, which, combined with PYTHIA's model for junction fragmentation [68], furnishes a new source of baryon production. Moreover, since it is the global colour structure of the event which gives rise to these additional baryons, their correlations (and net baryon-antibaryon compensation) can happen over longer distances than is the case for the conventional (local) baryon-production mechanisms in the string model.

In both cases, the fragmentation parameters of the string model are constrained by LEP data $[60,66]$. In particular, this means that the strangeness fraction is essentially fixed, modulo phase-space constraints and potential trigger-bias effects. The same is true for baryon production in the baseline CR model, while, in the New CR model, the baryon fraction can increase with the amount of CR. Exotic heavy baryons can also be formed, containing multiple charm or bottom quarks.

\subsection{DIPSY and rope hadronisation}

DIPSY [69] implements Mueller's dipole cascade model [70] which operates in transverse coordinate space (unlike conventional showers which operate in momentum space). Instead of conventional PDFs, the model starts from an explicit representation of each proton being composed of three colour dipoles in impact-parameter space and rapidity. These are then evolved in rapidity space via iterated gluon emission, forming a dipole cascade. The resulting partonic final states are hadronised using the Lund string model, via an interface to PYTHIA 8 [31].

The recently developed rope extension to DIPSY [71] allows geometrically nearby strings to act as a combined "colour rope", which can hadronise with a higher intrinsic tension. The consequences are larger $p_{\perp}$ kicks (relative to the string direction, which for soft-particle production in $p p$ collisions largely coincides with the $z$-axis) and production of more strange hadrons and baryons, the latter via probabilistic collapses of ropes to string junctions. The model also incorporates a mechanism called final-state "swing", which acts to minimise the masses of final-state colour-dipoles via colour reconnections, driven by SU (3) colour rules combined with an ad hoc probabilistic evolution kernel.

To provide a reference case without these effects, we also include a "NoSwing" tune of DIPSY with parameters optimised without use of the final-state rope and swing mechanisms.

\subsection{EPOS and hydrodynamic core hadronisation}

The EPOS MC [72] takes a Parton-Based Gribov Regge theory approach to event generation [73]. String hadronisation in EPOS is treated differently based on the local density of string segments per unit volume with respect to a critical-density parameter. Each string is classified as being in either a low density coronal region or in a high density core region.

Corona hadronisation proceeds via unmodified string fragmentation whereas the core is subjected to a hydrodynamic evolution; i.e. it is hadronised including additional contributions from longitudinal and radial flow effects [74]. Core conditions are easily satisfied in ion collisions, however even for an average $p p$ collision $\left(N_{\mathrm{ch}}=30,|\eta|<2.4\right)$ at $\sqrt{s}=7 \mathrm{TeV}$, around $30 \%$ of central particle production arises from the core region. This rises to $75 \%$ for $N_{\mathrm{ch}}=100$.

The EPOS LHC [75] tune considered here is a dedicated parameter set used to describe all LHC energies and incident particles. A loss of particle multiplicity due to radial flow rescaling is a feature needed to model ion collisions; however, such effects are not observed in $p p$ collisions. To compensate, the LHC tune adds an additional parametrisation which modifies the radial flow of the small, dense cores typical of $p p$ interactions to fix the cluster mass and hence maintain the particle multiplicity. The total momentum is then rescaled after the radial boost to preserve energy conservation.

\section{Observables}

Fiducial cuts are applied to the MC generator output to approximate experimental sensitivity and this results in an inclusive set of particles formed of two components. The 'prompt charged' component of the inclusive set consists of charged particles with $p_{\perp}>200 \mathrm{MeV},|\eta|<2.5$, lifetime $\tau>300 \mathrm{ps}$ and which are not created from the decay of a state with $30<\tau<300 \mathrm{ps}$. This set is dominantly $\pi^{ \pm}, K^{ \pm}$, $p$ and $\bar{p}$. The definition is based around the ATLAS fiducial selection in Ref. [76]. The second component consists of 'identifiable prompt strange hadrons'; here both charged and neutral strange hadrons are included if they typically undergo weak decay to one or more charged particles. These states are also required to satisfy $p_{\perp}>200 \mathrm{MeV},|\eta|<2.5$ and for themselves to not be created from the decay of other states with $30<\tau<300 \mathrm{ps}^{4}{ }^{4}$ This set is comprised of $K_{\mathrm{s}}^{0}$, $\Lambda, \bar{\Lambda}, \Xi^{ \pm}, \Sigma^{ \pm}, \bar{\Sigma}^{ \pm}$and $\Omega^{ \pm}$.

Track jets are clustered from prompt charged and prompt identifiable strange hadrons. They are reconstructed with the anti- $k_{t}$ algorithm [77] using radius parameter $R=0.4$, the leading jet is required to be within $|\eta|<2.3$.

\subsection{Underlying-event observables}

Standard Underlying Event nomenclature is used with respect to the leading track jet in the event as illustrated in Fig. 1. In this note we will consider quantities constructed

\footnotetext{
${ }^{4}$ So for example $\Lambda$ originating from $\Xi^{-}$decay are excluded.
} 


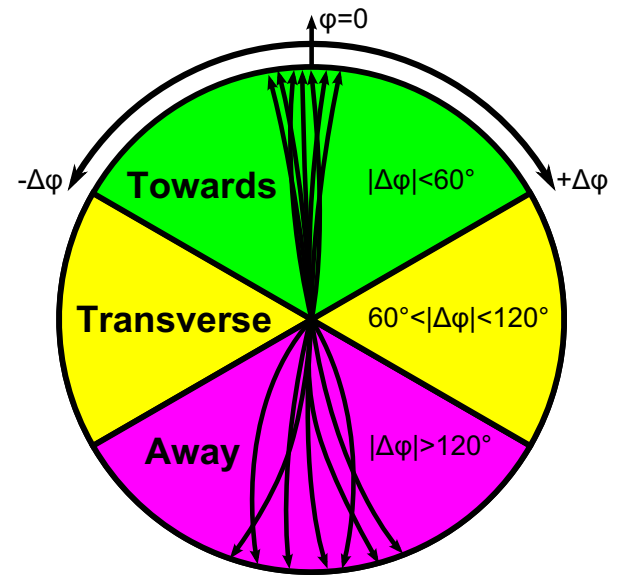

Fig. 1 Illustration of the towards, transverse and away regions of the azimuth with respect to the leading track jet in the event.

from particles only in the transverse region as this region is least affected by contributions from the leading $2 \rightarrow 2$ hard scatter.

The following variables are considered within the transverse region, these are common variables used to parametrise the underlying event as they probe the activity level and both the size and the shape of the particle $p_{\perp}$ distribution. The \langle\rangle notation implies an average over all events:

- $\left\langle N_{\text {Inc. }}\right\rangle$ : The event-averaged multiplicity of the inclusive set of particles (prompt charged plus prompt identifiable strange hadrons).

- $\langle N(X)\rangle$ : The event-averaged multiplicity of identified particle(s), $X$.

- $\left\langle\sum p_{\perp}\right\rangle$ : The event-averaged scalar sum of the transverse momentum of the inclusive set of particles.

- $\left\langle\right.$ mean $\left.p_{\perp}\right\rangle$ : The event-averaged mean $p_{\perp}$ of the inclusive set of particles.

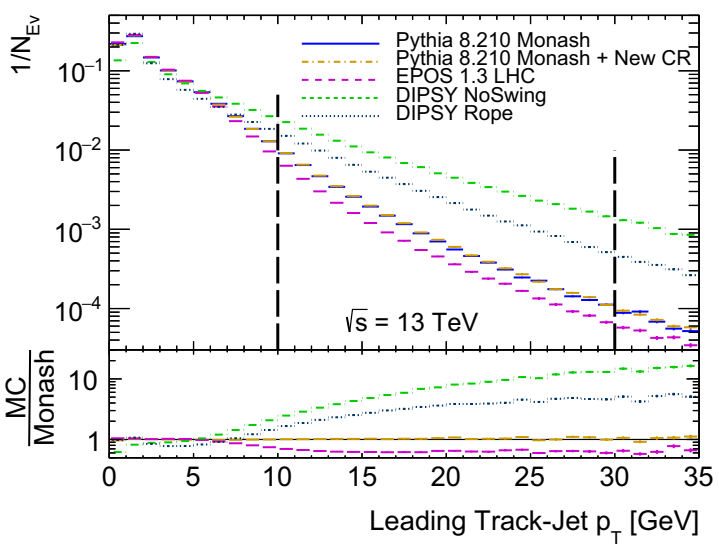

(a)
- $\langle$ RMS $\rangle$ : The event-averaged root mean square $p_{\perp}$ of the inclusive set of particles, defined here for a given multi-

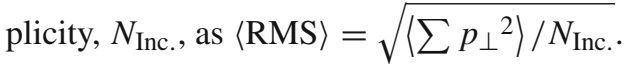

\subsection{Relative transverse activity classifier, $R_{\mathrm{T}}$}

Above a lower threshold corresponding to the onset of the UE plateau in the transverse region (roughly, $p_{\perp \text { lead }}>10 \mathrm{GeV}$ ), the mean values of UE quantities calculated over the full inclusive set of particles have little dependence on the leading track-jet $p_{\perp}$ : the multiple soft scatters which contribute the majority of the UE are largely independent of the leading jet. The slow rise of the UE plateau in the transverse region is understood to be due to additional contributions from wideangle radiation associated with the hard scatter, but this effect becomes significant only for jet $p_{\perp}>50 \mathrm{GeV}$ [23]. In this study, we focus on events just above the onset of the plateau, with $10 \mathrm{GeV}<p_{\perp \text { lead }}<30 \mathrm{GeV}$.

The shape of the leading track-jet $p_{\perp}$ spectrum in the various MC generators considered is shown in Fig. 2a. Dashed vertical lines indicate the lead $p_{\perp}$ range used in this study. The DIPSY tunes clearly exhibit a significantly harder jet $p_{\perp}$ spectrum than PYTHIA 8 or EPOS. However, when we consider the average inclusive-particle yields in the transverse region, shown in Fig. 2b, we observe that the activity in the transverse region is roughly constant and quite comparable between the models, independently of the jet $p_{\perp}$ spectrum. We therefore stress that any mis-modelling in the absolute rate of jet events is expected to have little effect on the results in this study due to the profiled mean normalisation. Note that the most extreme variant of DIPSY NOSWING does exhibit a delayed onset of the $\left\langle N_{\text {Inc. }}\right\rangle$ plateau. In the context of an experimental analysis, the robustness of the conclusions could be explicitly validated by comparing with one or

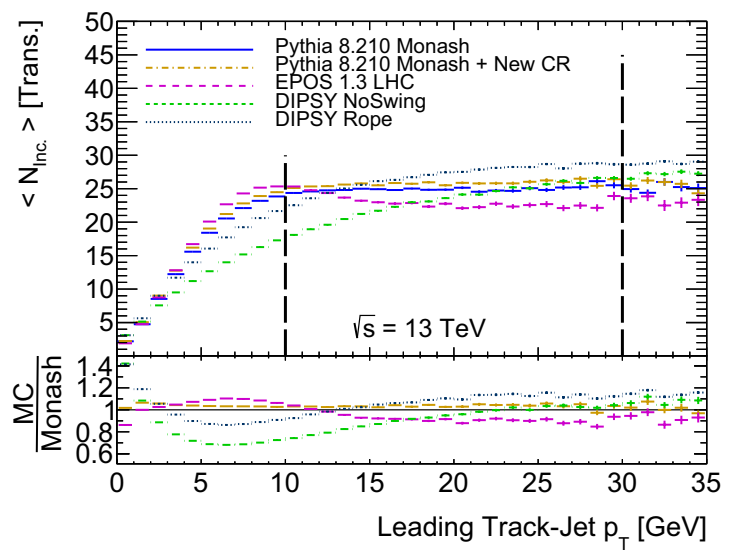

(b)

Fig. 2 Leading track-jet $p_{\perp}$ spectrum, normalised to unity (a) and $\left\langle N_{\text {Inc. }}\right\rangle$ in the transverse region as a function of leading track-jet $p_{\perp}$. Dashed vertical lines indicate the $p_{\perp}$ range used in this paper 


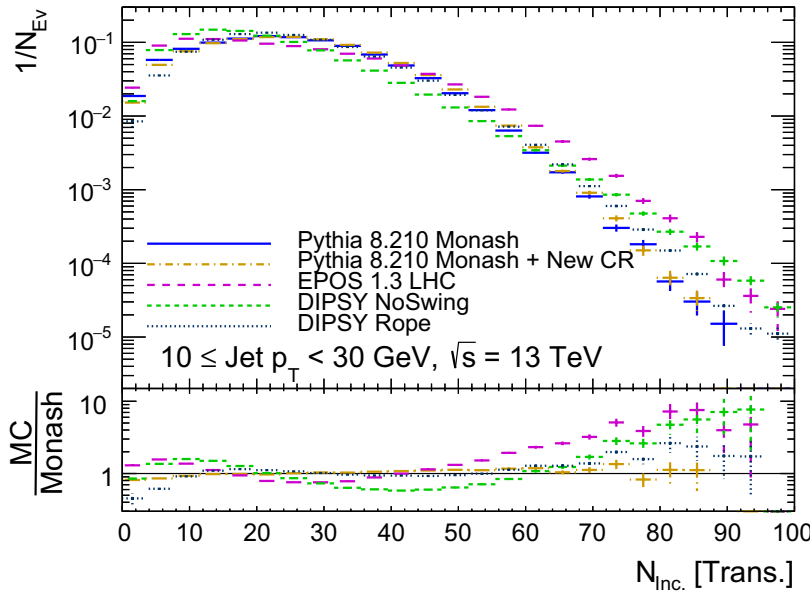

Fig. 3 The transverse $N_{\text {Inc. }}$ distribution for events with leading track jet in the range $10 \leq p_{\perp} 30<\mathrm{GeV}$, normalised to unity

more higher jet $p_{\perp}$ windows, say $20-40$ and/or $30-50 \mathrm{GeV}$, statistics allowing.

Although average quantities like $\left\langle N_{\text {Inc. }}\right\rangle$ in Fig. $2 \mathrm{~b}$ do not vary considerably with $p_{\perp}$ over this range of jet energies, the distributions underlying these mean values can be very broad. The full probability distribution of $N_{\text {Inc. }}$ is shown in Fig. 3, integrated over all events with leading track-jet $p_{\perp}$ values in the range $10-30 \mathrm{GeV}$. Such very broad distributions with tails much wider than Gaussians $\left(\Gamma \gg \sqrt{\left\langle N_{\text {Inc. }}\right\rangle}\right)$ are typical in minimum-bias and underlying-event studies. They imply that there is a very large dynamic range between transverseregion activities significantly larger or smaller than the mean. Given that the approximately constant average UE level is by now well established, we believe that these extremes, which are typically hidden in studies of 'average' UE properties, are the next natural focus of study.

In order to obtain an axis which allows for the investigation of the modelling of proton interactions as a function of the event activity, we reclassify events with a leading track jet in the range $10 \leq p_{\perp}<30 \mathrm{GeV}$ based on their per-event transverse activity with respect to the mean:

$R_{\mathrm{T}}=\frac{N_{\text {Inc. }}}{\left\langle N_{\text {Inc. }}\right\rangle}$.

We find this normalisation choice (which in minimum-bias contexts is referred to as the $\mathrm{KNO}$ variable [78]) to be useful since $R_{\mathrm{T}}=1$ then cleanly divides events with "higher-thanaverage" UE from "lower-than-average" ones, irrespective of $\mathrm{CM}$ energy or applied cuts. We note, however, that an absolute normalisation would be the preferred choice for determining, e.g., whether events with a fixed number of particles behave the same at all $\mathrm{CM}$ energies.

For each MC model, the value of the denominator in Eq. (1) corresponds to the mean values of the distributions in
Table 2 The average fiducial $\left\langle N_{\text {Inc. }}\right\rangle$, the denominator of $R_{\mathrm{T}}$, for the MC models considered along with the width of the distributions

\begin{tabular}{llll}
\hline Generator & Tune & $\left\langle N_{\text {Inc. }}\right\rangle$ & $\sigma$ \\
\hline PYTHIA 8 & Monash & 24.7 & 12.5 \\
PYTHIA 8 & Monash + New CR & 25.5 & 12.6 \\
EPOS & LHC & 24.2 & 14.6 \\
DIPSY & NoSwing & 21.3 & 12.2 \\
DIPSY & Rope & 25.1 & 12.0 \\
\hline
\end{tabular}

Fig. 3, which are tabulated in Table 2. All models predict a mean transverse multiplicity in the range $21-26$ and a width of around 13 (where Poissonian fluctuations would predict a much smaller width, $\sim \sqrt{25}=5$ ).

Measuring UE quantities versus $R_{\mathrm{T}}$ yields sensitivity to rare events with exceptionally large or small transverse activity with respect to the average event. The lower requirement on the leading track-jet $p_{\perp}$ acts to suppress soft-periphery and diffractive interactions by ensuring that a hard scatter was present while the upper requirement limits the contamination by wide-angle radiation off the hard scatter which increases slowly with the $p_{\perp}$ of the leading jet [23].

In the context of MPI models, the case where only a small amount of energy is deposited in the transverse region implies that only a small number of MPI occurred in that event. This therefore affords an opportunity to measure event properties in an 'MPI-suppressed' environment, where fragmentation properties may be closer to those of $e^{+} e^{-}$collisions than the average $p p$ jet event.

In the other extreme where the activity in an event is many times larger than the mean, new dynamic effects may become significant. Some different models which may give rise to modified behaviour in dense $p p$ interactions were discussed in Sect. 2. Such modifications may be important in describing effects already observed in data such as [56,79] and [49], and it is important to develop further probes that shed light on how these effects develop as we move from dilute to dense environments.

Illustrated in Fig. 4 are the average number of partonparton interactions (MPI) and the average transverse protonproton impact parameter $\left\langle b_{\mathrm{MPI}}\right\rangle$, with the latter normalised such that 1 corresponds to the impact parameter of an average minimum-bias event. Both are plotted as a function of $\log _{10}\left(R_{\mathrm{T}}\right)$ for four different tunes of PYTHIA 8. For the most active events $\left(\log _{10}\left(R_{\mathrm{T}}\right)=0.5\right.$ corresponding to $R_{\mathrm{T}}=\sqrt{10} \sim 3.2$ times higher-than-average UE activity) the average number of MPI increases by roughly a factor 2 relative to the mean (at $\log _{10}\left(R_{\mathrm{T}}\right)=0$ ), and the events are roughly twice as central as the average in this jet $p_{\perp}$ window (which in turn are twice as central as the average minimumbias event). For low-activity events, with less than a tenth of the average UE activity, $\log _{10}\left(R_{\mathrm{T}}\right)<-1$, an average of less 


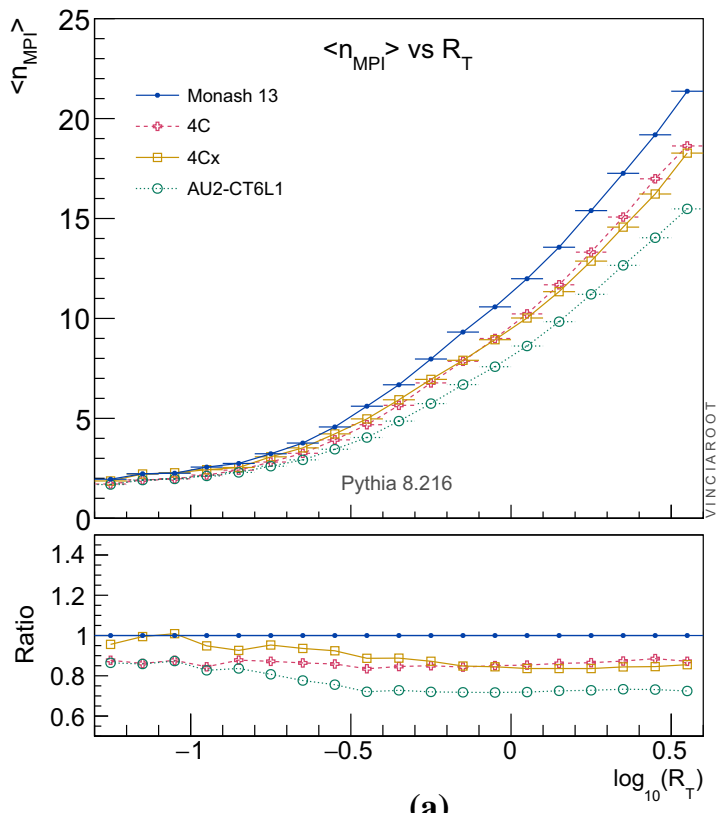

(a)

Fig. 4 The dependence of a the average number of parton-parton interactions (including the hardest interaction, hence $\left\langle n_{\mathrm{MPI}}\right\rangle \geq 1$ ) and $\mathbf{b}$ the average hadron-hadron impact parameter versus the transverse activity

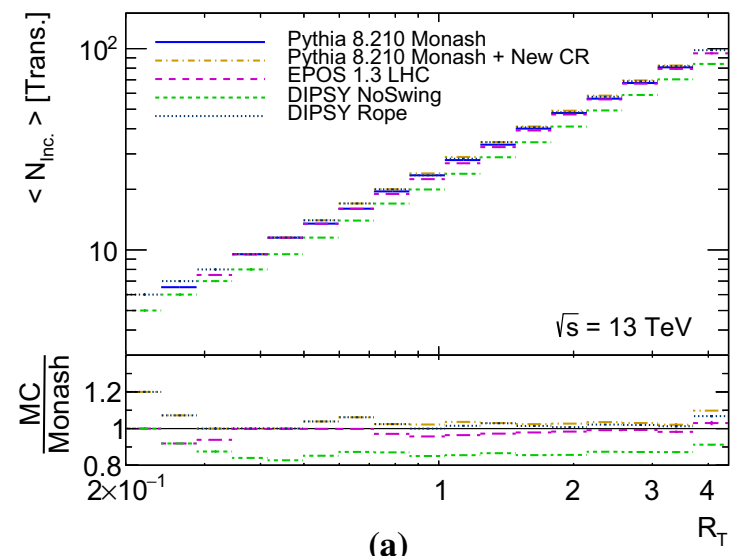

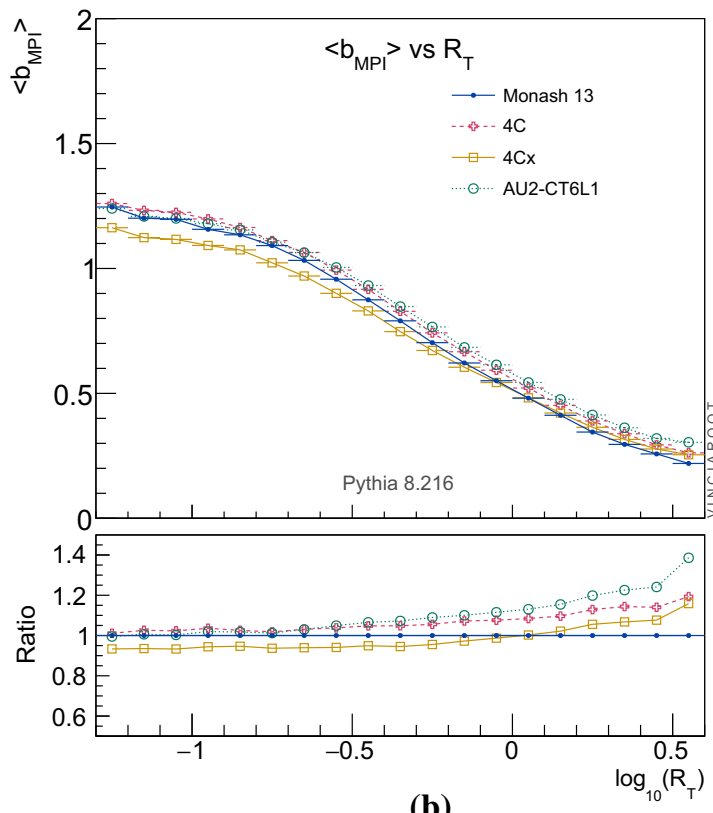

(b)

fraction, $R_{\mathrm{T}}$, defined in the text, for a representative set of PYTHIA 8 tunes, for $p p$ collisions at $13 \mathrm{TeV}$ with at least one track jet (defined in text)

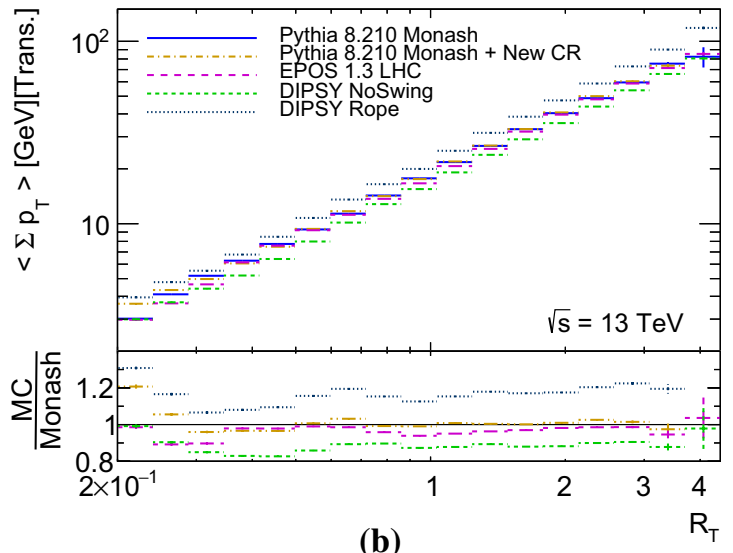

(b)

Fig. 5 Average multiplicity (a) of the set of inclusive particles and sum $p_{\perp}$ (b) as a function of the per-event activity classifier $R_{\mathrm{T}}$. All ratios are relative to PYTHIA 8.210 Monash

than 2 MPI per event are predicted by these models, with an average impact parameter even larger than for minimumbias events, $\left\langle b_{\mathrm{MPI}}\right\rangle>1$ for $\log _{10}\left(R_{\mathrm{T}}\right)<-0.5$. According to these models, our axis thus allows us to scan over almost an order of magnitude in both the average number of MPI and the average impact parameter, for fixed jet $p_{\perp}$.

For completeness, we note that relative UE activity could also have been classified using summed transverse-region $p_{\perp}$ or by using the jet median/area techniques referred to in the introduction. Our choice of $N_{\text {Inc. was }}$ based on two factors: firstly, we have a very direct relation with charged-particle multiplicity based minimum-bias studies of similar quantities, and secondly the fact that heavier hadrons exhibit harder $p_{\perp}$ spectra can lead to undesirable biases in a classifier based on $\sum p_{\perp}$. For example a low $\sum p_{\perp} \mathrm{UE}$ would be biased to be made up entirely of pions since these have the softest spectra. Such biases would complicate the interpretation of effects we wish to study such as any evolution in total strangeness and baryon fractions.

\section{Inclusive-particle set results}

As a precursor to the main study of identified-particle ratios, we first consider the evolution of the inclusive-particle spectra with $R_{\mathrm{T}}$. In Fig. 5, MC predictions for the average $N_{\text {Inc. }}$ 

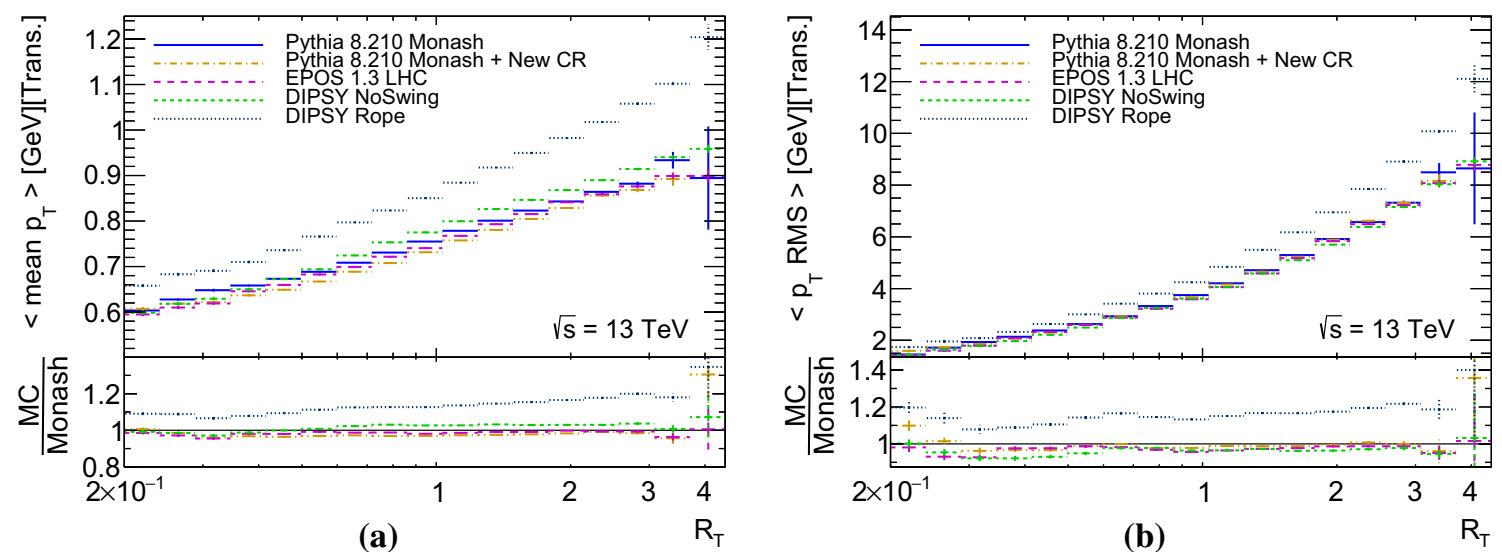

Fig. 6 Average mean (a) and RMS (b) of the $p_{\perp}$ distribution of the set of inclusive particles. All ratios are relative to PYTHIA 8.210 Monash

and $\sum p_{\perp}$ of the inclusive set of particles are plotted as a function of $R_{\mathrm{T}}$. The averages and widths of the inclusive $p_{\perp}$ spectra are investigated in Fig. 6. The DIPSY ROPE tune generates events with the highest overall average energy density followed by EPOS and PYTHIA 8. For DIPSY ROPE this is observed to be due to a similar average particle multiplicity as for PYTHIA 8 combined with a $20 \%$ harder mean $p_{\perp}$ distribution and a similarly larger RMS. The DIPSY NOSwING tune has both the lowest average multiplicity spectra and the energy density of the models, its mean $p_{\perp}$ is similar to that of PYTHIA 8.

Only minor differences are observed for PYTHIA 8 with the new colour-reconnection model with respect to the standard Monash tune in these distributions.

\section{Identified-particle results}

The production of identified particles is useful to investigate the evolution of the underlying event as a function of transverse activity levels in the event. The strange and baryon contents of the final state arising from non-perturbative effects are expected to be particularly sensitive to the modelling described in Sect. 2.

We normalise distributions to factor out any contribution from differences in the overall multiplicity spectra of the generators and focus on ratios of particle yields, with the total yield of inclusive particles discussed in the previous section. Crucially, this also highlights any changes in the relative suppression of identified particles, with respect to the inclusive sum. The meson fractions are plotted in Fig. 7. The ratio of charged pions to inclusive particles is given first as the latter is used subsequently for normalisation as it is experimentally easier to access. The $\pi^{ \pm}$fraction is observed to fall from $76-79 \%$ at low $R_{\mathrm{T}}$ to $72-77 \%$ at high $R_{\mathrm{T}}$, with the lowest fractions in EPOS and DIPSY ROPE at high $R_{\mathrm{T}}$ as expected due to increased strange and baryon production in these models as a function of $R_{\mathrm{T}}$. PYTHIA 8 Monash + New CR, which does not incorporate strangeness enhancement but does allow for baryon enhancement, also exhibits a statistically significant drop.

The average $K_{\mathrm{s}}^{0}$ and $K^{ \pm}$multiplicities with respect to the inclusive multiplicity are shown in Fig. 7b, c. A baseline increase in the kaon fraction with $R_{\mathrm{T}}$ due to phase-space effects is observed for the PYTHIA 8 Monash and DIPSY NOSWING tunes. EPOS predicts a significant strange meson enhancement at high $R_{\mathrm{T}}$ whereas DIPSY ROPE predict an enhancement for all $R_{\mathrm{T}}$, in both cases it reaches 5-10\%. Interestingly PYTHIA 8 Monash + new CR exhibits slightly lower charged- and neutral-kaon fractions at all $R_{\mathrm{T}}$. This is likely to be due to a smaller baseline strangeness fraction in that tune combined with a tendency of the model to create a large number of low-mass string systems in which the production of high-mass hadrons (including strange ones) is suppressed due to phase-space restrictions.

When the ratio of the doubly strange $\phi(s \bar{s})$ meson multiplicity is taken with respect to that of the singly strange $K_{\mathrm{s}}^{0}$ in Fig. 7d, the new CR model in PYTHIA 8 does not produce additional $\phi$ and is also in agreement with DIPSY NOSwING. Both DIPSY ROPE and EPOS however, which both incorporate explicit strangeness enhancement, are shown to produce considerably more $\phi$ on average, up to $30 \%$ for DIPSY ROPE and $75 \%$ for EPOS with respect to PYTHIA 8.

The baryonic enhancement is probed in Fig. 8. In Fig. 8a the $p \bar{p}$ multiplicity is normalised to the inclusive charged multiplicity. Some phase-space effects are apparent in all MCs with the smallest rise in proton enhancement coming from PYTHIA 8 and DIPSY NOSWING. In EPOS the magnitude of the enhancement is steepest as a function of $R_{\mathrm{T}}$ leading it to be in agreement with PYTHIA 8 and DIPSY NOSwING only for low values of $R_{\mathrm{T}}$. PYTHIA $8+$ new CR and DIPSY ROPE both predict significant enhancement, with up to a $50 \%$ 


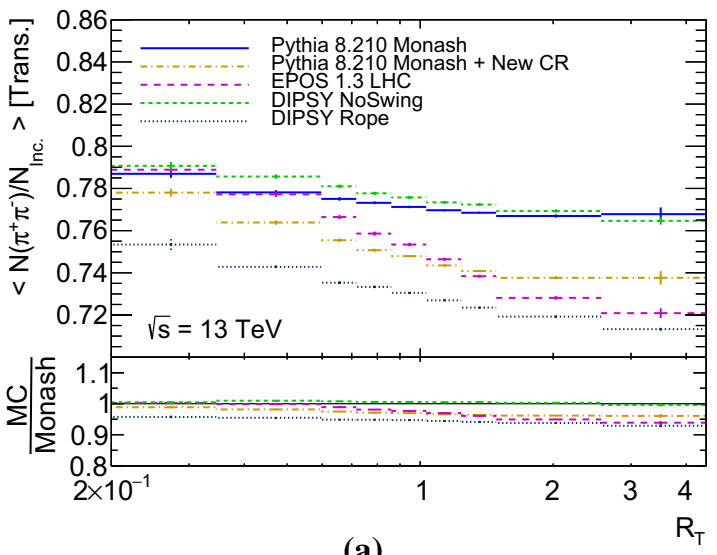

(a)

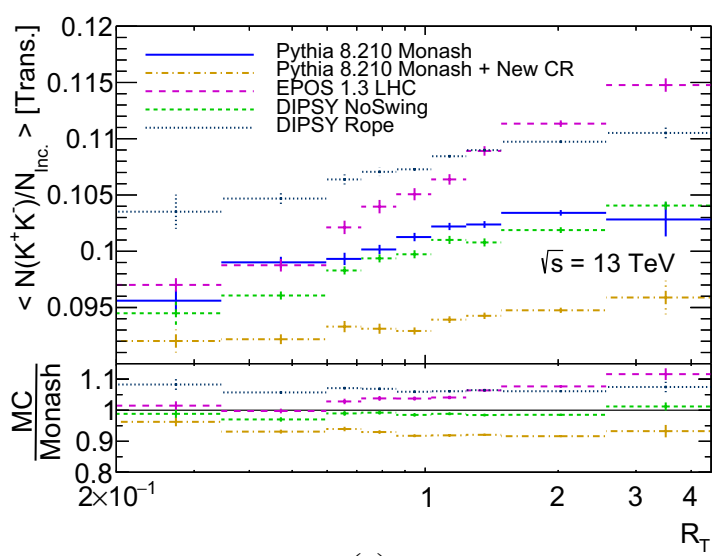

(c)

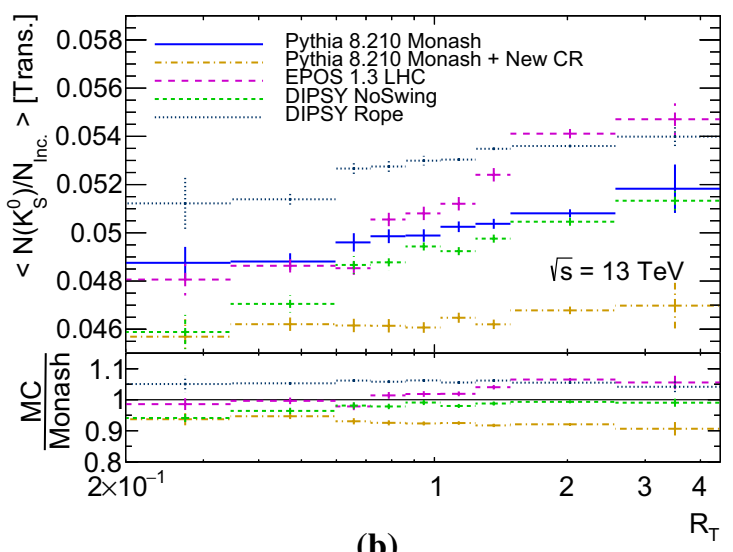

(b)

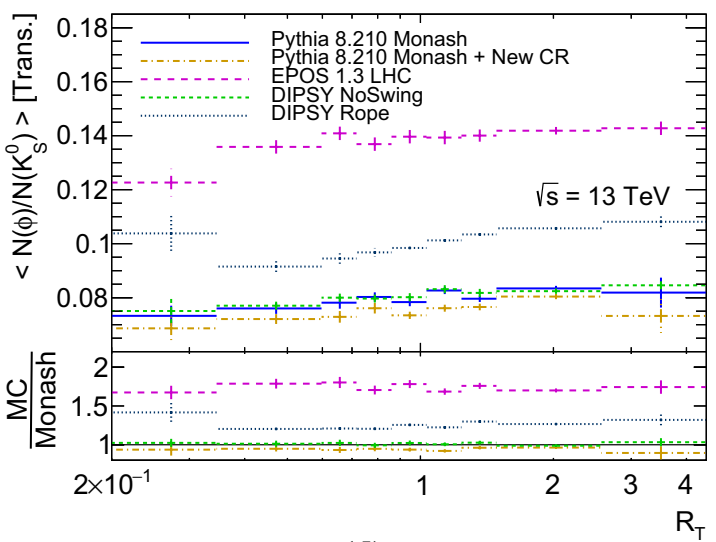

(d)

Fig. 7 Normalised average identified-meson yields in the transverse region as a function of $R_{\mathrm{T}}$ for $\pi^{+} \pi^{-} / N_{\text {Inc. }}(\mathbf{a}), K_{\mathrm{s}}^{0} / N_{\text {Inc. }}(\mathbf{b}), K^{+} K^{-} / N_{\text {Inc. }}$. (c) and $\phi / K_{\mathrm{S}}^{0}(\mathbf{d})$. Shown for different MC models and tunes. All ratios are relative to PYTHIA 8.210 Monash

increase in proton fraction relative to their simpler model tunes.

By normalising the proton yield to the charged-kaon yield, in Fig. 8b, we obtain a probe that is sensitive to any relative enhancement between baryons and strangeness. PYTHIA 8 Monash + new CR exhibits the highest ratio, as expected since it incorporates a mechanism for baryon enhancement but no mechanism for strangeness enhancement. Next highest is DIPSY ROPE, due to a baryon enhancement significantly larger than its strange meson enhancement. We note that in EPOS, the two effects are of the same magnitude in both sectors-making it similar to the baseline models, PYTHIA 8 and DIPSY NOSWING in this observable.

In Fig. 8c strange-baryon enhancement is plotted in the ratio of $\Lambda \bar{\Lambda}$ hyperons to $K_{\mathrm{s}}^{0}$. The profile is similar to that of the proton enhancement except that all of EPOS, DIPSY ROPE and PYTHIA $8+$ new $\mathrm{CR}$ are in reasonable agreement and show up to a $90 \%$ enhancement effect with increasing $R_{\mathrm{T}}$.

Finally doubly strange $\Xi^{ \pm}$baryons are investigated in Fig. 8d normalised to the $\Lambda \bar{\Lambda}$ multiplicity spectra. A clear enhancement with increasing $R_{\mathrm{T}}$ is observed only for EPOS; other models do not predict a strong rise; however, DIPSY ROPE predicts a slightly larger baseline fraction of doubly strange to singly strange baryons.

\section{Conclusions}

A new axis for underlying-event studies $R_{\mathrm{T}}=N_{\text {Inc. }} /\left\langle N_{\text {Inc. }}\right\rangle$ is proposed to be used as an alternate to leading-object $p_{\perp}$ for events which contain at least one hard scatter and are hence on the 'underlying-event plateau'. $R_{\mathrm{T}}$ isolates events with exceptionally large or small activity in the transverse underlying-event region with respect to the event-averaged mean. Distributions which are sensitive to hadronisation effects are studied as a function of this event-activity classifier.

Identified-particle ratios (strangeness and in particular baryon fractions) are observed to be highly sensitive to the event activity density with a marked increase in average $p \bar{p} / N_{\text {Inc. }}$ and $\Lambda \bar{\Lambda} / K_{\mathrm{s}}^{0}$ multiplicity ratios in dense events for PYTHIA 8 with the new colour-reconnection model, DIPSY 


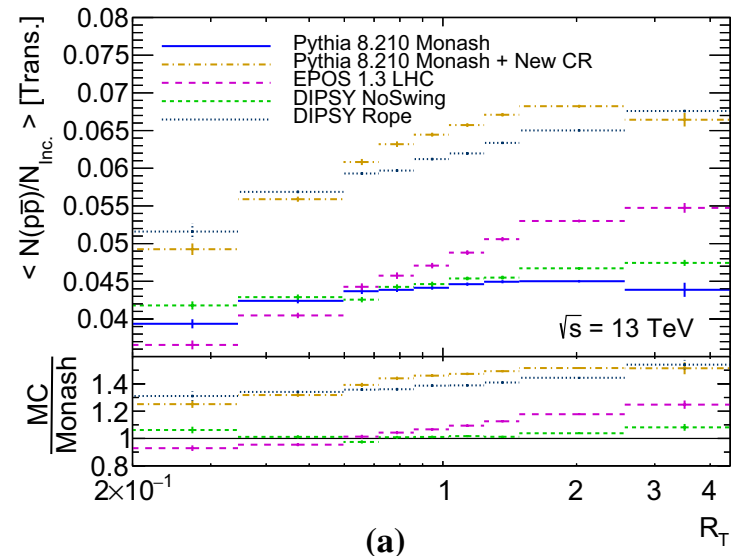

(a)

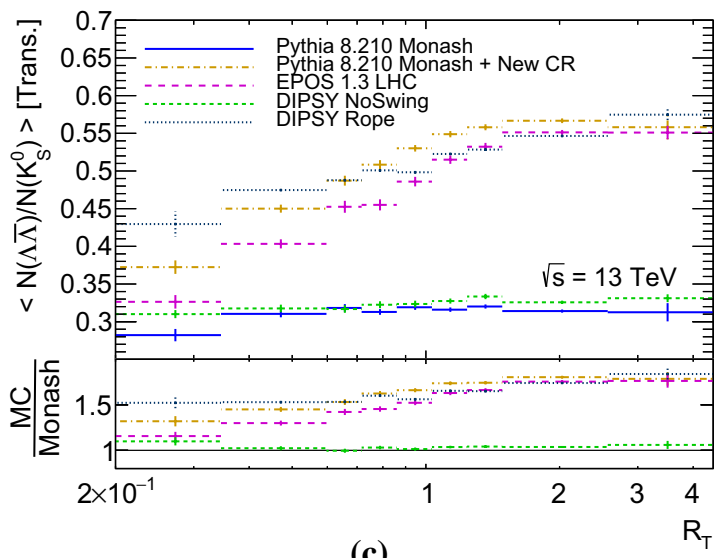

(c)
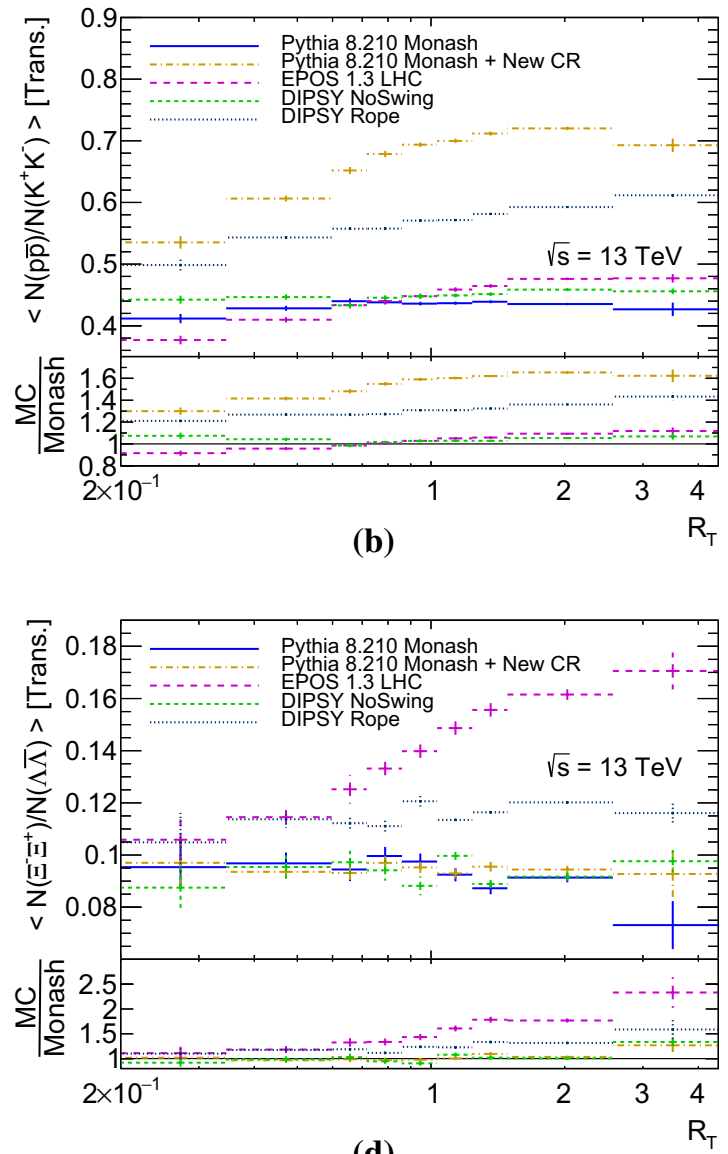

(d)

Fig. 8 Normalised average identified-baryon yields in the transverse region as a function of $R_{\mathrm{T}}$ for $p \bar{p} / N_{\text {Inc. }}(\mathbf{a}), p \bar{p} / K^{+} K^{-}(\mathbf{b}), \Lambda \bar{\Lambda} / K_{\mathrm{S}}^{0}(\mathbf{c})$ and $\Xi^{+} \Xi^{-} / \Lambda \bar{\Lambda}$ (d). Shown for different MC models and tunes. All ratios are relative to PYTHIA 8.210 Monash

ROPE and EPOS when compared to models with a less detailed treatment, PYTHIA 8 and DIPSY NOSWING.

Distributions are also presented for $K, \phi$ and $\Xi^{ \pm}$resonances.

These distributions are experimentally accessible at the LHC and would allow for discrimination between alternate colour-reconnection, colour "rope" and hydrodynamic modelling of dense proton-proton interactions.

Acknowledgments We gratefully acknowledge support from the Monash-Warwick Alliance Development Fund, without which this study would not have been possible. PS is the recipient of an Australian Research Council Future Fellowship, FT130100744. This work was also supported in part by the ARC Centre of Excellence for Particle Physics at the Terascale and we acknowledge the support of the UK Science and Technology Facilities Council. We thank C. Bierlich and J. Christiansen for supplying the tunes of DIPSY and PYTHIA 8 used in this paper.

Open Access This article is distributed under the terms of the Creative Commons Attribution 4.0 International License (http://creativecomm ons.org/licenses/by/4.0/), which permits unrestricted use, distribution, and reproduction in any medium, provided you give appropriate credit to the original author(s) and the source, provide a link to the Creative
Commons license, and indicate if changes were made. Funded by SCOAP ${ }^{3}$.

\section{References}

1. UA1 Collaboration, G. Arnison et al., Hadronic jet production at the CERN proton - anti-proton collider. Phys. Lett. B 132, 214 (1983)

2. A. Buckley et al., General-purpose event generators for LHC physics. Phys. Rep. 504, 145-233 (2011). arXiv:1101.2599 [hepph]

3. UA1 Collaboration, G. Arnison et al., Transverse energy distributions in the central calorimeters. in Proceedings, 21st International Conference on High Energy Physics (ICHEP 1982), 1982. http:// alice.cern.ch/format/showfull?sysnb $=0050334$

4. UA1 Collaboration, G. Arnison et al., Some observations on the first events seen at the CERN proton-anti-proton collider. Phys. Lett. B 107, 320-324 (1981). [Erratum: Phys. Lett. 109B, 510(1982)]

5. UA1 Collaboration, G. Arnison et al., Charged particle multiplicity distributions in proton anti-proton collisions at $540 \mathrm{GeV}$ center-ofmass energy. Phys. Lett. B 123, 108 (1983)

6. UA1 Collaboration, C. Albajar et al., Production of low transverse energy clusters in anti-p p collisions at $\sqrt{s}=0.2 \mathrm{TeV}$ to $0.9 \mathrm{TeV}$ 
and their interpretation in terms of QCD jets. Nucl. Phys. B 309, 405 (1988)

7. STAR Collaboration, H. Caines, Exploring jet properties in p-p collisions at $200 \mathrm{GeV}$ with STAR. Nucl. Phys. A 830, 263C-266C (2009). arXiv:0907.3460 [nucl-ex]

8. STAR Collaboration, H. Caines, Jet and underlying event measurements in $\mathrm{p}+\mathrm{p}$ collisions at RHIC. Nucl. Phys. A 855, 376-379 (2011)

9. CDF Collaboration, T. Affolder et al., Charged jet evolution and the underlying event in $p \bar{p}$ collisions at $1.8 \mathrm{TeV}$. Phys. Rev. D 65, 092002 (2002)

10. CDF Collaboration, D. Acosta et al., The underlying event in hard interactions at the Tevatron $\bar{p} p$ collider. Phys. Rev. D 70, 072002 (2004). arXiv:hep-ex/0404004 [hep-ex]

11. CDF Collaboration, T. Aaltonen et al., Studying the underlying event in drell-yan and high transverse momentum jet production at the tevatron. Phys. Rev. D 82, 034001 (2010). arXiv:1003.3146 [hep-ex]

12. CDF Collaboration, T. Aaltonen et al., A study of the energy dependence of the underlying event in proton-antiproton collisions. arXiv:1508.05340 [hep-ex]

13. CMS Collaboration, V. Khachatryan et al., First measurement of the underlying event activity at the LHC with $\sqrt{s}=0.9 \mathrm{TeV}$. Eur. Phys. J. C 70, 555-572 (2010). arXiv:1006.2083 [hep-ex]

14. ATLAS Collaboration, G. Aad et al., Measurement of underlying event characteristics using charged particles in pp collisions at $\sqrt{s}=900 \mathrm{GeV}$ and $7 \mathrm{TeV}$ with the ATLAS detector. Phys. Rev. D 83, 112001 (2011). arXiv:1012.0791 [hep-ex]

15. ATLAS Collaboration, G. Aad et al., Measurements of underlyingevent properties using neutral and charged particles in $p p$ collisions at $900 \mathrm{GeV}$ and $7 \mathrm{TeV}$ with the ATLAS detector at the LHC," Eur. Phys. J. C71 (2011) 1636, arXiv:1103.1816 [hep-ex]

16. CMS Collaboration, S. Chatrchyan et al., Measurement of the underlying event activity at the LHC with $\sqrt{s}=7 \mathrm{TeV}$ and comparison with $\sqrt{s}=0.9$ TeV. JHEP 09, 109 (2011). arXiv:1107.0330 [hep-ex]

17. ALICE Collaboration, B. Abelev et al., Underlying event measurements in $p p$ collisions at $\sqrt{s}=0.9$ and $7 \mathrm{TeV}$ with the ALICE experiment at the LHC. JHEP 07, 116 (2012). arXiv:1112.2082 [hep-ex]

18. ATLAS Collaboration, G. Aad et al., Measurements of the pseudorapidity dependence of the total transverse energy in proton-proton collisions at $\sqrt{s}=7 \mathrm{TeV}$ with ATLAS. JHEP 11, 033 (2012). arXiv:1208.6256 [hep-ex]

19. CMS Collaboration, S. Chatrchyan et al., Measurement of the underlying event activity in $p p$ collisions at $\sqrt{s}=0.9$ and $7 \mathrm{TeV}$ with the novel jet-area/median approach. JHEP 08, 130 (2012). arXiv:1207.2392 [hep-ex]

20. CMS Collaboration, S. Chatrchyan et al., Measurement of the underlying event in the Drell-Yan process in proton-proton collisions at $\sqrt{s}=7$ TeV. Eur. Phys. J. C 72, 2080 (2012). arXiv:1204.1411 [hep-ex]

21. CMS Collaboration, S. Chatrchyan et al., Study of the underlying event at forward rapidity in pp collisions at $\sqrt{s}=0.9,2.76$, and 7 TeV. JHEP 04, 072 (2013). arXiv:1302.2394 [hep-ex]

22. CMS Collaboration, S. Chatrchyan et al., Jet and underlying event properties as a function of charged-particle multiplicity in protonproton collisions at $\sqrt{s}=7$ TeV. Eur. Phys. J. C 73(12), 2674 (2013). arXiv: 1310.4554 [hep-ex]

23. ATLAS Collaboration, G. Aad et al., Measurement of the underlying event in jet events from $7 \mathrm{TeV}$ proton-proton collisions with the ATLAS detector. Eur. Phys. J. C 74(8), 2965 (2014). arXiv:1406.0392 [hep-ex]

24. ATLAS Collaboration, G. Aad et al., Measurement of distributions sensitive to the underlying event in inclusive Z-boson production in $p p$ collisions at $\sqrt{s}=7 \mathrm{TeV}$ with the ATLAS detector. Eur. Phys. J. C 74(12), 3195 (2014). arXiv:1409.3433 [hep-ex]

25. CMS Collaboration, V. Khachatryan et al., Measurement of the underlying event activity using charged-particle jets in protonproton collisions at $\sqrt{s}=2.76 \mathrm{TeV}$. JHEP 09, 137 (2015). arXiv:1507.07229 [hep-ex]

26. ATLAS Collaboration, G. Aad et al., Leading track underlying event at $13 \mathrm{TeV}$. https://cds.cern.ch/record/2037684

27. M. Cacciari, G.P. Salam, S. Sapeta, On the characterisation of the underlying event. JHEP 04, 065 (2010). arXiv:0912.4926 [hep-ph]

28. M. Heinrich, A jet based approach to measuring soft contributions to proton-proton collisions with the CMS experiment. $\mathrm{PhD}$ thesis, KIT, Karlsruhe, EKP, 2011. https://inspirehep.net/record/ 1087971/files/CERN-THESIS-2011-190.pdf

29. C. Bierlich, J.R. Christiansen, Effects of color reconnection on hadron flavor observables. Phys. Rev. D 92(9), 094010 (2015). arXiv:1507.02091 [hep-ph]

30. M. Bähr et al., Herwig++ physics and manual. Eur. Phys. J. C 58, 639-707 (2008). arXiv:0803.0883 [hep-ph]

31. T. Sjöstrand, S. Ask, J.R. Christiansen, R. Corke, N. Desai, P. Ilten, S. Mrenna, S. Prestel, C.O. Rasmussen, P.Z. Skands, An introduction to PYTHIA 8.2. Comput. Phys. Commun. 191, 159-177 (2015). arXiv:1410.3012 [hep-ph]

32. T. Gleisberg, S. Hoeche, F. Krauss, M. Schonherr, S. Schumann, F. Siegert, J. Winter, Event generation with SHERPA 1.1. JHEP 02, 007 (2009). arXiv:0811.4622 [hep-ph]

33. A. Karneyeu, L. Mijovic, S. Prestel, P.Z. Skands, MCPLOTS: a particle physics resource based on volunteer computing. Eur. Phys. J. C 74, 2714 (2014). arXiv:1306.3436 [hep-ph]

34. CDF Collaboration, R. Field, R.C. Group, PYTHIA tune A, HERWIG, and JIMMY in run 2 at CDF. arXiv:hep-ph/0510198 [hep-ph]

35. T. Sjöstrand, M. van Zijl, A multiple interaction model for the event structure in hadron collisions. Phys. Rev. D 36, 2019 (1987)

36. T. Sjöstrand, S. Mrenna, P.Z. Skands, PYTHIA 6.4 physics and manual. JHEP 05, 026 (2006). arXiv:hep-ph/0603175 [hep-ph]

37. L.D. McLerran, M. Kataja, P.V. Ruuskanen, H. von Gersdorff, Studies of the hydrodynamical evolution of matter produced in fluctuations in $\mathrm{p}$ anti-p collisions and in ultrarelativistic nuclear collisions. 2. Transverse momentum distributions. Phys. Rev. D 34, 2755 (1986)

38. UA1 Collaboration, C. Albajar et al., A study of the general characteristics of $p \bar{p}$ collisions at $\sqrt{s}=0.2 \mathrm{TeV}$ to $0.9 \mathrm{TeV}$. Nucl. Phys. B 335, 261-287 (1990)

39. CDF Collaboration, D. Acosta et al., Soft and hard interactions in $p \bar{p}$ collisions at $\sqrt{s}=1800 \mathrm{GeV}$ and $630 \mathrm{GeV}$. Phys. Rev. D 65, 072005 (2002)

40. CDF Collaboration, T. Aaltonen et al., Measurement of particle production and inclusive differential cross sections in $p \bar{p}$ collisions at $\sqrt{s}=1.96-$ TeV. Phys. Rev. D 79, 112005 (2009). arXiv:0904.1098 [hep-ex]. [Erratum: Phys. Rev. D 82, 119903 (2010)]

41. ATLAS Collaboration, G. Aad et al., Charged-particle multiplicities in pp interactions measured with the ATLAS detector at the LHC. New J. Phys. 13, 053033 (2011). arXiv:1012.5104 [hep-ex]

42. CMS Collaboration, V. Khachatryan et al., Charged particle multiplicities in $p p$ interactions at $\sqrt{s}=0.9,2.36$, and $7 \mathrm{TeV}$. JHEP 01, 079 (2011). arXiv:1011.5531 [hep-ex]

43. STAR Collaboration, B.I. Abelev et al., Strange baryon resonance production in $\sqrt{s}(\mathrm{NN})=200 \mathrm{GeV} \mathrm{p}+\mathrm{p}$ and $\mathrm{Au}+$ Au collisions. Phys. Rev. Lett. 97, 132301 (2006). arXiv:nucl-ex/0604019 [nucl-ex]

44. CDF Collaboration, T. Aaltonen et al., Production of $\Lambda, \bar{\Lambda}^{0} \Xi^{ \pm}$ and $\Omega^{ \pm}$hyperons in $p \bar{p}$ collisions at $\sqrt{s}=1.96 \mathrm{TeV}$. Phys. Rev. D 86, 012002 (2012). arXiv:1101.2996 [hep-ex]

45. ATLAS Collaboration, G. Aad et al., Kshort and $\Lambda$ production in $p p$ interactions at $\sqrt{s}=0.9$ and $7 \mathrm{TeV}$ measured with the ATLAS detector at the LHC," Phys. Rev. D 85 (2012) 012001, arXiv:1111.1297 [hep-ex] 
46. CMS Collaboration, V. Khachatryan et al., "Strange Particle Production in $p p$ Collisions at $\sqrt{s}=0.9$ and 7 TeV. JHEP 05, 064 (2011). arXiv:1102.4282 [hep-ex]

47. LHCb Collaboration, R. Aaij et al., "Measurement of prompt hadron production ratios in $p p$ collisions at $\sqrt{s}=0.9$ and $7 \mathrm{TeV}$. Eur. Phys. J. C 72, 2168 (2012). arXiv:1206.5160 [hep-ex]

48. ALICE Collaboration, B. Abelev et al., Multi-strange baryon production in $p p$ collisions at $\sqrt{s}=7 \mathrm{TeV}$ with ALICE. Phys. Lett. B 712, 309-318 (2012). arXiv:1204.0282 [nucl-ex]

49. CMS Collaboration, S. Chatrchyan et al., Measurement of neutral strange particle production in the underlying event in protonproton collisions at $\sqrt{s}=7$ TeV. Phys. Rev. D 88, 052001 (2013). arXiv:1305.6016 [hep-ex]

50. ALICE Collaboration, B. B. Abelev et al., Production of $\Sigma(1385)^{ \pm}$ and $\Xi(1530)^{0}$ in proton-proton collisions at $\sqrt{s}=7 \mathrm{TeV}$. Eur. Phys. J. C 75(1), 1 (2015). arXiv:1406.3206 [nucl-ex]

51. STAR Collaboration, B.I. Abelev et al., Strange particle production in $\mathrm{p}+\mathrm{p}$ collisions at $\sqrt{s}=200 \mathrm{GeV}$. Phys. Rev. C 75, 064901 (2007). arXiv:nucl-ex/0607033 [nucl-ex]

52. ALICE Collaboration, K. Aamodt et al., Production of pions, kaons and protons in $p p$ collisions at $\sqrt{s}=900 \mathrm{GeV}$ with ALICE at the LHC. Eur. Phys. J. C 71, 1655 (2011). arXiv:1101.4110 [hep-ex]

53. ALICE Collaboration, B. Abelev et al., Production of $K^{*}(892)^{0}$ and $\phi(1020)$ in $p p$ collisions at $\sqrt{s}=7$ TeV. Eur. Phys. J. C 72, 2183 (2012). arXiv:1208.5717 [hep-ex]

54. ALICE Collaboration, J. Adam et al., Measurement of pion, kaon and proton production in proton-proton collisions at $\sqrt{s}=7 \mathrm{TeV}$. Eur. Phys. J. C 75(5), 226 (2015). arXiv:1504.00024 [nucl-ex]

55. CMS Collaboration, V. Khachatryan et al., Observation of longrange near-side angular correlations in proton-proton collisions at the LHC. JHEP 09, 091 (2010). arXiv:1009.4122 [hep-ex]

56. ATLAS Collaboration, G. Aad et al., Observation of long-range elliptic anisotropies in $\sqrt{s}=13$ and $2.76 \mathrm{TeV} p p$ collisions with the ATLAS detector. arXiv:1509.04776 [hep-ex]

57. CDF Collaboration, D. Acosta et al., $K_{S}^{0}$ and $\Lambda^{0}$ production studies in $p \bar{p}$ collisions at $\sqrt{s}=1800 \mathrm{GeV}$ and $630 \mathrm{GeV}$. Phys. Rev. D 72, 052001 (2005). arXiv:hep-ex/0504048 [hep-ex]

58. ALICE Collaboration, B. Abelev et al., Multiplicity dependence of two-particle azimuthal correlations in pp collisions at the LHC. JHEP 09, 049 (2013). arXiv:1307.1249 [nucl-ex]

59. A. Ortiz, G. Bencédi, H. Bello, S. Jena, Jet effects in highmultiplicity pp events. in 7th International Workshop on Multiple Partonic Interactions at the LHC (MPI@LHC 2015) Miramare, Trieste, Italy, November 23-27, 2015. 2016. arXiv:1603.05213 [hep-ph]

60. P. Skands, S. Carrazza, J. Rojo, Tuning PYTHIA 8.1: the Monash 2013 Tune. Eur. Phys. J. C 74(8), 3024 (2014). arXiv:1404.5630 [hep-ph]

61. A. Buckley, J. Butterworth, L. Lonnblad, D. Grellscheid, H. Hoeth, J. Monk, H. Schulz, F. Siegert, Rivet user manual. Comput. Phys. Commun. 184, 2803-2819 (2013). arXiv:1003.0694 [hep-ph]

62. M. Cacciari, G.P. Salam, G. Soyez, FastJet user manual. Eur. Phys. J. C 72, 1896 (2012). arXiv:1111.6097 [hep-ph]
63. T. Sjöstrand, P.Z. Skands, Transverse-momentum-ordered showers and interleaved multiple interactions. Eur. Phys. J. C 39, 129-154 (2005). arXiv:hep-ph/0408302 [hep-ph]

64. T. Sjöstrand, P.Z. Skands, Multiple interactions and the structure of beam remnants. JHEP 03, 053 (2004). arXiv:hep-ph/0402078 [hep-ph]

65. S. Argyropoulos, T. Sjöstrand, Effects of color reconnection on $t \bar{t}$ final states at the LHC. JHEP 11, 043 (2014). arXiv:1407.6653 [hep-ph]

66. J.R. Christiansen, P.Z. Skands, String formation beyond leading colour. JHEP 08, 003 (2015). arXiv:1505.01681 [hep-ph]

67. R. Corke, T. Sjöstrand, Interleaved parton showers and tuning prospects. JHEP 03, 032 (2011). arXiv:1011.1759 [hep-ph]

68. T. Sjöstrand, P.Z. Skands, Baryon number violation and string topologies. Nucl. Phys. B 659, 243 (2003). arXiv:hep-ph/0212264 [hep-ph]

69. C. Flensburg, G. Gustafson, L. Lönnblad, Inclusive and exclusive observables from dipoles in high energy collisions. JHEP 08, 103 (2011). arXiv:1103.4321 [hep-ph]

70. A.H. Mueller, Soft gluons in the infinite momentum wave function and the BFKL pomeron. Nucl. Phys. B 415, 373-385 (1994)

71. C. Bierlich, G. Gustafson, L. Lönnblad, A. Tarasov, Effects of overlapping strings in pp collisions. JHEP 03, 148 (2015). arXiv:1412.6259 [hep-ph]

72. K. Werner, F.-M. Liu, T. Pierog, Parton ladder splitting and the rapidity dependence of transverse momentum spectra in deuterongold collisions at RHIC. Phys. Rev. C 74, 044902 (2006). arXiv:hep-ph/0506232 [hep-ph]

73. H.J. Drescher, M. Hladik, S. Ostapchenko, T. Pierog, K. Werner, Parton based Gribov-Regge theory. Phys. Rep. 350, 93-289 (2001). arXiv:hep-ph/0007198 [hep-ph]

74. K. Werner, Core-corona separation in ultra-relativistic heavy ion collisions. Phys. Rev. Lett. 98, 152301 (2007). arXiv:0704.1270 [nucl-th]

75. T. Pierog, I. Karpenko, J.M. Katzy, E. Yatsenko, K. Werner, EPOS LHC: test of collective hadronization with data measured at the CERN large hadron collider. Phys. Rev. C 92(3), 034906 (2015). arXiv:1306.0121 [hep-ph]

76. ATLAS Collaboration, G. Aad et al., Charged-particle distributions in $\sqrt{s}=13 \mathrm{TeV} p p$ interactions measured with the ATLAS detector at the LHC. arXiv:1602.01633 [hep-ex]

77. M. Cacciari, G.P. Salam, G. Soyez, The anti-k(t) jet clustering algorithm. JHEP 04, 063 (2008). arXiv:0802.1189 [hep-ph]

78. Z. Koba, H.B. Nielsen, P. Olesen, Scaling of multiplicity distributions in high-energy hadron collisions. Nucl. Phys. B 40, 317-334 (1972)

79. CMS Collaboration, S. Chatrchyan et al., Observation of longrange near-side angular correlations in proton-lead collisions at the LHC. Phys. Lett. B 718, 795-814 (2013). arXiv:1210.5482 [nucl-ex] 